\title{
Internet On-Ramp
}

\section{Snapping Up SNPs}

Stuart M. Brown, NYU Medical Center, New York, NY (browns02@mcrcr6.med.edu)

Single nucleotide polymorphisms (SNPs) are a hot item in current discussions about the practical applications of genomics and Human Genome Project research. SNPs are simply DNA point mutations-single base-pair changes or insertion/deletions-that are present at measurable frequencies in the population. SNPs are the most common variations in the human genome; the current estimate is that SNPs occur once every 100-300 bp. SNPs exist at defined positions within genomes (sequence tagged sites [STS]) and can be used for gene mapping, defining population structure and performing functional studies. SNPs are expected to greatly facilitate large-scale genetic studies concerned with determining linkage between sequence variations and heritable phenotypes. SNPs might also become an efficient tool for genetic identification for legal and forensic applications.

SNPs are particularly interesting as markers because many known genetic diseases, such as sickle cell anemia, are in fact caused by single base mutations. Therefore, an assay for an SNP marker could directly identify the disease-causing mutation. Some genetic diseases, such as cystic fibrosis, are caused by any of a large number of different mutations in a single gene. SNPs can be used to quickly assay for all known alleles in a large gene.

SNPs have been proposed as an ideal tool for the emerging discipline of pharmacogenomics. This new discipline hopes to use genetic information to fine-tune patient diagnosis and treatment. Large-scale analysis of the associations between the effects of drugs and genetic markers may allow physicians to match drugs to the genetic makeup of individual patients to better predict beneficial and harmful effects.

Some advantages of SNPs over other types of genetic markers such as isozymes, restriction fragment length polymorphisms, variable number tandem repeats and simple sequence repeats include: very large numbers of polymorphic loci; loci distributed throughout the genome; markers present within coding regions, introns and regions that flank genes; simple and unambiguous assay techniques; high levels of polymorphism in the population; stable Mendelian inheritance; and low levels of spontaneous mutation. Kwok et. al (2) have observed that most of the SNPs identified from sequence databases are highly heterozygous in the population.

\section{SNP Databases on the Web}

In January 1998, Francis Collins, director of the National Human Genome Research Institute (NHGRI), launched a $\$ 30$ million project to create a collection of 100000 SNP markers to be "calibrated" against a standard set of human DNA samples and made available to any interested scientist. In September 1998, the National Center for Biotechnology Information (NCBI) in collaboration with NHGRI established a database of SNPs (dbSNP) on its Web site (http://www.ncbi.nIm. nih.gov/SNP) to serve as a central repository of SNP sequences. This database contains sequence information flanking each polymorphism, links to related GenBank ${ }^{\circledR}$ accessions and to the GenBank database of STS (dbSTS), the experimental conditions used to assay the polymorphism and the frequency of alleles in various ethnic groups. The dbSNP has 3953 accessions (as of February 1999), and it is growing at a rate of 100-500 new entries per month.

The NHGRI and the Centers for Disease Control have developed a set of "standard" human DNA samples (NIH Polymorphism Discovery Resource [NIHPDR]) that can be used to evaluate polymorphic markers in a consistent manner and to facilitate comparisons of markers developed by different research groups using different methods. This resource will comprise cell lines and DNA from 450 unrelated individuals, female and male. It is designed to reflect the range of diversity in the human population. The material in the resource will be available to any investigator from the Coriell Institute for Medical Research (Camden, NJ, USA; http://arginine. umdnj.edu/).

The National Cancer Institute ( $\mathrm{NCl}$ ) has taken an alternate approach to assembling a database of useful SNP markers. The NCl project, know as the Genetic Annotation Initiative (GAI), looks for SNPs by sequencing the coding regions and flanking regions of several thousand genes suspected of contributing to cancer susceptibility or resistance. SNPs discovered by the GAI are added to the NCBl's public dbSNP. This will complement the NCl's Cancer Genome Anatomy project, which collects STS data from a broad set of cancerous and noncancerous tissues.

The Whitehead Institute/MIT Center for Genome Research, has a Human SNP Mapping Project funded by a consortium consisting of Affymetrix, Bristol-Myers Squibb and Millennium Pharmaceuticals. As of May 1998, they released data on 2227 mapped SNPs and 3358 total SNPs. These data are available on their Web site (http://www.genome.wi.mit.edu/SNP/human/ index.html). This Web site also includes a nice graphical map locating its SNP markers on all human chromosomes. 